\title{
Reliability Analysis of Tunnel Lining under Earthquake Loading Using Response Surface Method
}

\author{
Shuli Fan ${ }^{1, a}$, Yi Shi ${ }^{1, b}$, Dongyuan Sun ${ }^{2, c}$, Jianyun Chen ${ }^{1, d}$ \\ ${ }^{1}$ State Key Lab. of Coastal and Offshore Engineering, Dalian University of Technology, Dalian, China \\ ${ }^{2}$ Liaoning Provincial Transportation Planning and Design Institute, Shenyang, China \\ ashuli@dlut.edu.cn, bㅁagongshiyi@163.com, 로2885240@qq.com, deerd001@dlut.edu.cn
}

Keywords: Tunnel; Reliability analysis; Response surface method; Sensitivity

Abstract. The variation of rock mass mechanical parameters has a significant impact on the dynamic response of tunnel lining. Regarding the deformation modulus, Poisson's ratio, cohesion and frictional angle of rock mass as basic random variables, the effect of these parameters on the reliability index of tunnel ling was researched using finite element method. Response Surface Methodology was utilized to estimate the functional relationship between the load effects on tunnel lining and mechanical parameters based on numerical results. The results show that the reliability indices decrease with the means and variation coefficient of basic random variables increasing, and the mechanical parameters of rock mass have different sensitivities on the reliability index of tunnel lining in different situations.

\section{Introduction}

Generally, in aseismic design of tunnel, rock mass surrounding tunnel is considered as homogeneous material and the rock properties are assumed estimates from empirical characterization techniques or based on relevant data collected for other tunnel projects in similar area. More recently, probabilistic methods have been adopted to account for uncertainty in rock mass properties. Hang-Zhou Li and Bak Kong Low [1] regarded the cohesion, frictional angle and deformation modulus as normally distributed random variables and used the first-order reliability method (FORM) to calculated the reliability index of a circular tunnel. Qing Lü et al. [2] used the response surface method (RSM) to perform the reliability analysis of a circle tunnel and analyzed the failure probability with respect to different criteria. B.K. Low and H.H. Einstein [3] examined two existing definitions of factor of safety for a roof wedge in a tunnel and proposed a reliability-based design for tunnel supports. Liu Hui-jun and $\mathrm{Yu} \mathrm{Su}$ [4] analyzed the stress and deformation of tunnel lining over time and determined the safety of the tunnel lining structure. However, these reliability analyses of tunnels have not considered the dynamic response under earthquake loading.

In this study, the reliability of tunnel lining suffering earthquake was investigated considering the deformation modulus, Poisson's ratio, cohesion and frictional angle of rock mass as basic random variables. The discussion was focused on the effect of variation coefficient and mean value of rock mass parameters on the reliability index of tunnel lining under earthquake. At same time the sensitivity of these parameters were also investigated.

\section{Analysis of Loading Effect on Tunnel Lining}

Performance Function. In tunnel engineering projects, numerical simulation results show that the shearing force carried by tunnel lining is much less than the shear strength of concrete. The eccentric compression is the main reason of tunnel lining failure [5]. Following the standard convention from the code for design of road tunnel (JTG D70-2004), the performance function for tunnel lining can be defined as: 


$$
Z=\left\{\begin{array}{cc}
\varphi \alpha \sigma_{c} b h-N & e_{0} \leq 0.2 h \\
\frac{1}{6}\left(1.75 \varphi \sigma_{t} b h^{2}+h N\right)-M & e_{0} \geq 0.2 h
\end{array} .\right.
$$

Where $\varphi$ is longitudinal bending coefficient; $\alpha$ is eccentric effect factor; $\sigma_{c}$ is compressive strength of concrete; $\sigma_{t}$ is tensile strength of concrete; $b$ is longitudinal dimension, and assumed to be equal to 1 ; $h$ is the section thickness; $e_{0}=M / N ; N$ and $M$ are the axial force and bending moment of tunnel lining, respectively.

Response Surface Function of Load Effects. RSM has a wide application in civil engineering reliability analysis [6]. In RSM, the actual function is approximated, usually by a polynomial function. The finite element analysis is used to evaluate the function in the region where the design point is expected to be located on the actual (but implicit) function [2]. The resulting approximate, fitted surface then becomes the explicit equivalent of the implicit function.

For the case that the reliability assessment problem under consideration is governed by $n$ basic random variables $x_{1}, x_{2}, \ldots, x_{n}$. Using a second-order polynomial response surface function to fit the relationship between internal forces and basic random variables:

$$
Z=g\left(x_{1}, x_{2}, \ldots, x_{n}\right)=a+\sum_{i=1}^{n} b_{i} x_{i}+\sum_{i=1}^{n} c_{i} x_{i},
$$

where, $a, b_{i}, c_{i}$ are indeterminate coefficients; $x_{1}, x_{2}, \ldots, x_{n}$ are basic random variables.

In this study, the deformation modulus $E$, Poisson's ratio $\mu$, cohesion $c$ and frictional angle $\varphi$ of rock mass were regarded as basic random variables, other parameters are regarded as constant values. The rock mass mechanical properties and statistical characteristics were summarized in Table 1.

Table 1: Statistical characteristics of variables

\begin{tabular}{ccccc}
\hline Random Variables & $E(\mathrm{GPa})$ & $\mu$ & $c(\mathrm{MPa})$ & $\tan \varphi$ \\
\hline Mean value & 12 & 0.26 & 2 & 0.7 \\
\hline Standard deviation & 1.8 & 0.15 & 0.2 & 0.1 \\
\hline Coefficient of variation & 0.15 & 0.058 & 0.1 & 0.143 \\
\hline Distribution & Normal & Normal & Normal & Normal \\
\hline Variable $x_{i}$ & $x_{1}$ & $x_{2}$ & $x_{3}$ & $x_{4}$ \\
\hline
\end{tabular}

It was assumed that the axial force $N$ and bending moment $M$ of tunnel lining were the functions of $E, \mu, c$ and $\tan \varphi$. The functions can be approximated by a second-order model polynomial model according equation (2):

$$
\begin{aligned}
& M=a_{0}+a_{1} x_{1}+a_{2} x_{2}+a_{3} x_{3}+a_{4} x_{4}+a_{5} x_{1}^{2}+a_{6} x_{2}^{2}+a_{7} x_{3}^{2}+a_{8} x_{4}^{2}, \\
& N=b_{0}+b_{1} x_{1}+b_{2} x_{2}+b_{3} x_{3}+b_{4} x_{4}+b_{5} x_{1}^{2}+b_{6} x_{2}^{2}+b_{7} x_{3}^{2}+b_{8} x_{4}^{2},
\end{aligned}
$$

where, $x_{1}, x_{2}, x_{3}$ and $x_{4}$ are the deformation modulus $E$, Poisson's ratio $\mu$, cohesion $c$, and frictional angle $\varphi$ of rock mass respectively. $a_{0}, a_{1}, \ldots, a_{8}$ and $b_{0}, b_{1}, \ldots, b_{8}$ are regression coefficients. The regression coefficients are obtained from discrete evaluations of the implicit function.

A general method proposed by Gong Jinxin [7] for computing reliability index was used to calculate the reliability index of tunnel lining under seismic loading.

\section{Reliability Analysis of a Circle Tunnel Lining}

A Supported Tunnel and Its Parameters. The geometric characterized of the current tunnel was shown in Fig. 1(a). It is a horseshoe tunnel with height and span of $9.62 \mathrm{~m}$ and $12.22 \mathrm{~m}$, respectively. The lining is assumed to be $0.5 \mathrm{~m}$ thick. It is buried with the depth of $64 \mathrm{~m}$ from the top of the tunnel to ground surface.

As illustrated in Fig. 1(b), the rock mass around the tunnel was modeled with a 2D finite element model consisting of four-node quadrilateral elements. The tunnel lining was simulated using beam 
elements. In order to reduce or eliminate reflections of earthquake waves for boundaries of the finite element model, the infinite elements were added to the finite element model on the three sides of the model. The Mohr-Coulomb failure criterion was taken to simulate the plasticity of the rock mass and the lining is assumed as elastic. The statistical characteristics of rock parameters were summarized in Table 1. For tunnel lining, $E=24 \mathrm{GPa}, \mu=0.17$ and $\rho=2400 \mathrm{~kg} / \mathrm{m}^{3}$. The El Centro wave was selected as input excitation. The Peak Ground Acceleration (PGA) is 100gal. The attenuation coefficient is assumed to be $5 \%$.

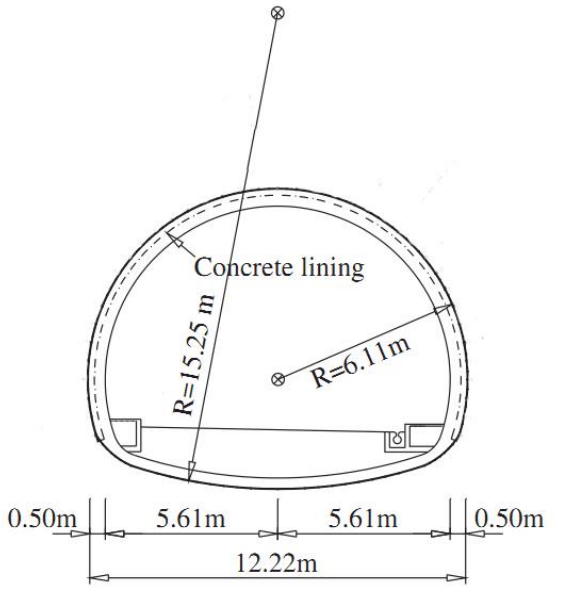

(a) cross-section of tunnel in geometry

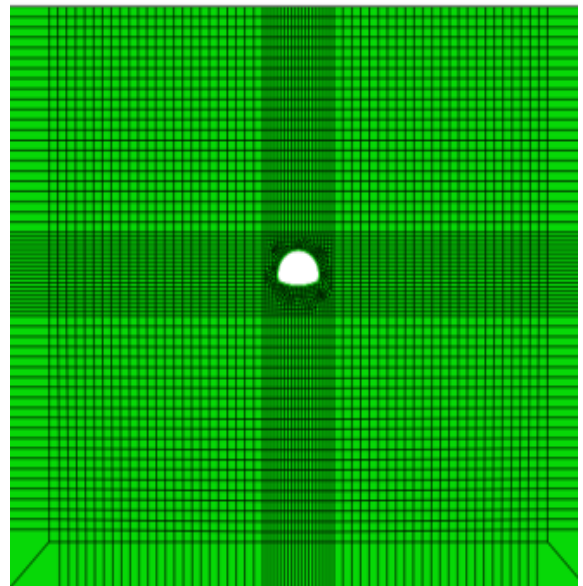

(b) finite element model

Fig. 1: The tunnel lining characteristics and finite element model

Reliability Index of Tunnel Lining Affected by Rock Parameters Variable Coefficient. For rock mass mechanical parameters, $E, \mu, c$ and $\varphi$, reasonable ranges are full of uncertainty. In order to clear the effect of these parameters on the reliability of tunnel lining, the reliability and sensitivity indices under earthquake loading were calculated to quantify the impact of variation coefficients and mean values of rock mass mechanical parameters on the reliability of tunnel lining.



Fig. 2: Changing curves of reliability index with varying of variation coefficient

Eight cases with different coefficients of every variation were calculated to investigate the reliability index variation. Coefficient varies from 0.05 to 0.4 . In these analyses, when one input variable regarded as random variables, other input variables are treated as deterministic inputs. Fig. 2 shows the trend of the effect of variation coefficient on the reliability index. The reliability indices decrease significantly with the increase of variation coefficients when the mean values of $E, \mu$ and $c$ are determined. This indicate that the reliability index has a great change with a small change of the variation coefficients. When the variation coefficient is 0.05 , the reliability index is 12.15 . It is dramatically reduced to 3.55 when the variation coefficient is 0.4 , decreasing $68.5 \%$. When the variation coefficients are greater than 
0.05, the declining tendency of reliability indices becomes slower. For the parameter $\tan \varphi$, the reliability index is almost constant when the variation coefficient ranges from 0.05 to 0.2 . However, the reliability index presents drastically decreasing tendency when it is greater than 0.2 .



(a) effect of $E$ mean value

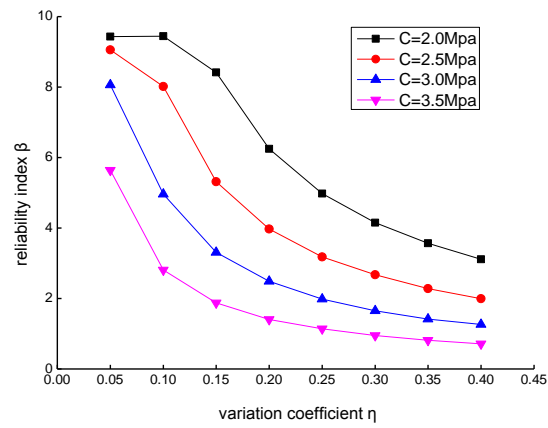

(c) effect of $c$ mean value



(b) effect of $\mu$ mean value

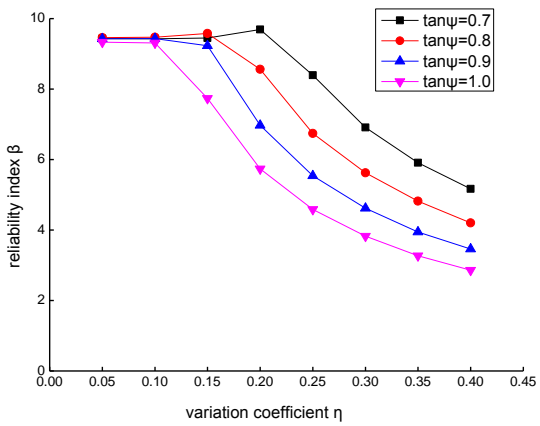

(d) effect of $\tan \varphi$ mean value

Fig. 3: Changing curves of reliability index with varying of mean value

Fig. 3 shows the change curves of reliability index with the increasing of variation coefficient when $\mathrm{s}$ the mean value of rock mass mechanical parameters increases. The reliability index increase with the same coefficient of variation. When the mean value of $E$ increases from $12 \mathrm{GPa}$ to $15 \mathrm{GPa}$, the reliability index decreases except the case that the variation coefficient equals to 0.05 . When the mean value of deformation modulus $E$ is $15 \mathrm{GPa}$, the index decreases about $74.8 \%$ with the variation increasing to 0.4 from 0.1 . As for parameter $\mu$ and $c$, the reliability indices of tunnel lining also decline with the mean value increasing in different values of variation coefficient. The reliability index is almost stability by changing the mean value of $\tan \varphi$ while the variation coefficient increases to 0.1 from 0.05 .

Sensitivity analysis. An approximation of the sensitivity of reliability index can be determined for each parameter $x$ using the following equation [8]:

$$
S_{\beta}=\frac{\Delta \beta}{\Delta x} \bullet \frac{x_{0}}{\beta_{0}} \text {. }
$$

Where $S_{\beta}$ is the sensitivity of reliability index, $x_{0}$ is a constant of the variable $x$ in basic case, $\beta_{0}$ is the corresponding reliability index when $x$ takes $x_{0}$.

The basic case, with the properties of rock mass listed in Table 1, is applied to calculate the sensitivity of reliability to changes in random variables for cases with different variation coefficient and mean value. Corresponding, the reliability index $\beta_{0}$ at the crown of liner equal to 9.443 in the basic case.

In the sensitivity analysis of the effect of variation coefficient, variation coefficient of $E, \mu, c$ and $\tan \varphi$ were treated as a random variable to reflect its uncertainty, and the mean values of these 
parameters were regarded as constants. As seen in Fig. 4, the sensitivity of rock mass mechanical parameters on reliability index increases with the variation coefficient $\eta$. The variability of $\mu$ has a larger impact on the sensitivity of reliability index than other rock mass mechanical parameters. When the coefficient of variation is 0.4 , the sensitivity of Poisson's ratio $\mu$ increases to 5 while the sensitivities of the cohesion $c$ and deformation modulus $E$ are about 2.7. The sensitivity of friction coefficient $\tan \varphi$ increases slowly with the variation coefficient changing. When the coefficient of variation is 0.4 , the sensitivity of friction coefficient $\tan \varphi$ is just 1.286 .

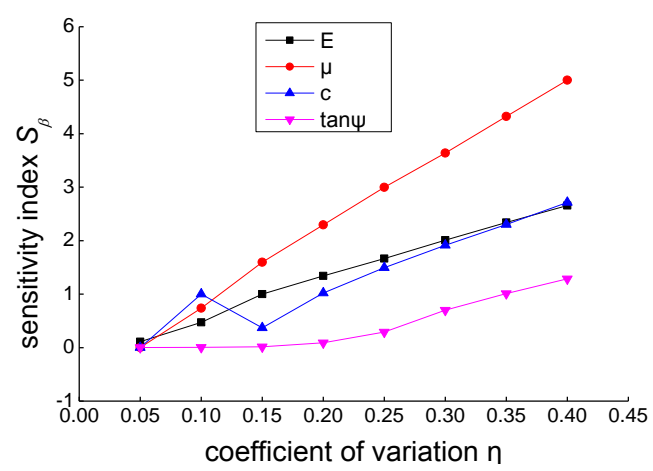

Fig. 4: The sensitivity indexes of reliability under surrounding rock parameter variation

Table 2: The sensitivity indexes $S_{E}$ under the mean change of $E$

\begin{tabular}{ccccccccc}
\hline$E(\mathrm{Gpa})$ & 11.5 & 12 & 12.5 & 13 & 13.5 & 14 & 14.5 & 15 \\
\hline$\beta_{E}$ & 7.59 & 7.07 & 6.60 & 6.14 & 5.74 & 5.35 & 4.98 & 4.66 \\
\hline$S_{E}$ & 1.74 & 1.77 & 1.80 & 1.94 & 1.89 & 2.04 & 2.11 & 2.07 \\
\hline
\end{tabular}

Table 3: The sensitivity indexes $S_{\mu}$ under the mean change of $\mu$

\begin{tabular}{ccccccccc}
\hline$\mu$ & 0.25 & 0.26 & 0.27 & 0.28 & 0.29 & 0.3 & 0.31 & 0.32 \\
\hline$\beta_{\mu}$ & 3.92 & 3.59 & 3.26 & 2.96 & 2.68 & 2.45 & 2.20 & 1.97 \\
\hline$S_{\mu}$ & 2.44 & 2.38 & 2.71 & 2.84 & 3.00 & 2.91 & 3.49 & 3.74 \\
\hline
\end{tabular}

Table 4: The sensitivity indexes $S_{c}$ under the mean change of $c$

\begin{tabular}{ccccccccc}
\hline$c(\mathrm{MPa})$ & 2.0 & 2.2 & 2.4 & 2.6 & 2.8 & 3 & 3.2 & 3.4 \\
\hline$\beta_{c}$ & 6.25 & 5.21 & 4.36 & 3.64 & 3.01 & 2.49 & 2.01 & 1.59 \\
\hline$S_{c}$ & 2.06 & 2.19 & 2.35 & 2.55 & 2.93 & 3.13 & 3.81 & 4.45 \\
\hline
\end{tabular}

Table 5: The sensitivity indexes $S_{\tan \varphi}$ under the mean change of $\tan \varphi$

\begin{tabular}{ccccccccc}
\hline $\tan \varphi$ & 0.5 & 0.6 & 0.7 & 0.8 & 0.9 & 1 & 1.1 & 1.2 \\
\hline$\beta_{\tan \varphi}$ & 8.96 & 9.16 & 9.69 & 8.56 & 6.97 & 5.74 & 4.75 & 3.94 \\
\hline$S_{\tan \varphi}$ & 0.09 & 0.13 & 0.38 & 1.05 & 2.05 & 2.15 & 2.28 & 2.46 \\
\hline
\end{tabular}

Table 2 to Table 5 show the sensitivity of rock mass mechanical parameters on reliability index changing with the mean value of parameter. For deformation modulus $E$ of rock mass, the fluctuation of sensitivity index is stability, it means that the reliability index has less affected by the mean value of $E$. For $\mu$ and $c$, the sensitivity indexes obviously increase with the changing of their means, hence the mean values of $\mu$ and $c$ have an essential impact on reliability index. However, the sensitivity index is small and changes smoothly when the mean of $\tan \varphi$ ranges from 0.5 to 0.7 , which means the reliability index is stability. The sensitivity index gradually increases as the mean value of $\tan \varphi$ changes from 0.7 to 1.2 . The reliability index decreases rapidly when the mean value of $\tan \varphi$ is greater than 0.7 . So it has a giant effect on reliability index. 


\section{Summary and Conclusions}

Comparing with results, the contents and conclusions can be summarized as follows:

(1) With the increasing of variation coefficient, the reliability index of tunnel lining is trending downward. But for the parameter $\tan \varphi$, the reliability index is almost constant when the variation coefficient ranges from 0.05 to 0.2 .

(2) When the mean value of $E, \mu, c$ and $\tan \varphi$ changes, the variation tendencies of reliability indices are almost the same. With the increasing of mean value, the reliability index is on the decrease. But the decreasing extents are different when the mean values increase with a certain step length.

(3) The variability of $\mu$ has a larger impact on the sensitivity of reliability index than other rock mass mechanical parameters. The reliability index has less affected by the mean value of $E$. For $\mu$ and $c$, the sensitivity indexes obviously increase with the changing of their means.

\section{Acknowledgments}

This research was financially supported by the National Natural Science Foundation of China (Grant NO. 51178081) and Chinese Scholarship Council (CSC).

\section{References}

[1] Hang-Zhou Li, Bak Kong Low. Reliability analysis of circular tunnel under hydrostatic stress field[J]. Computers and Geotechnics, 2010,37(1-2):50-58.

[2] Lü Qing, Sun Hong-Yue, Bak Kong Low. Reliability analysis of ground-support interaction in circular tunnels using the response surface method[J]. International Journal of Rock Mechanics and Mining Sciences, 2011,48(8):1329-1343.

[3] Bak Kong Low, H.H. Einstein. Reliability analysis of roof wedges and rockbolt forces in tunnels[J]. Tunnelling and Underground Space Technology, 2013,38:1-10.

[4] Liu Hui-jun, Yu Su: "Stress and deformation analysis of lining in tunneling process[J]. The Electronic Journal of Geotechnical Engineering”[J] 2014(19.U):6273-6281.

[5] G. Besnard, X. Garros, F. Andrieu, et al. Superior performance and Hot Carrier reliability of strained FDSOI nMOSFETs for advanced CMOS technology nodes[J]. Solid-State Electronics, 2015, 113:127-131.

[6] Guan X. L., R.E. Melchers. Effect of response surface parameter variation on structural reliability estimates[J]. Structural Safety, 2002,23(2001):429-444.

[7] Gong Jinxin, Zhong Weiqiu, Zhao Guofan. A general approach for computing structural reliability index[J]. Chinese Journal of Computational Mechanics, 2003,20(1):12-18.

[8] Wu Guojun, Chen Weizhong, Liu Doudou, Tan Xianjun. Reliability analysis of tunnel lining based on continuum model[J]. Rock and Soil Mechanics, 2006,24(S1):359-363. 